\section{AN UNUSUAL CASE OF EPISTAXIS IN A WHITE TIGER (PANTHERA TIGRIS)}

\section{Nath ${ }^{1}$, V.S.C. Bose ${ }^{1}$, T.K. Pattanaik ${ }^{1}$, S.K. Panda ${ }^{2}$, R.K. Samantray ${ }^{3}$ and P.K. Roy ${ }^{3}$}

\author{
${ }^{1}$ Associate Professor Department of Surgery, ${ }^{2}$ Assistant \\ Professor, Department of Pathology, Orissa Veterinary College, \\ Bhubaneswar, Orissa 751003, India \\ ${ }^{3}$ Veterinary Officer, Nandankanan Zoo, Orissa, India
}

web supplement

At Nandankanan Zoo a white tigress Shreeya, aged 17 years and weighing about $160 \mathrm{~kg}$ was detected to be anorectic on 17.xi.04. She was treated with cefotaxime sodium $1 \mathrm{~g}$ (Taxim Inj.; Alkem Laboratory Ltd., Mumbai) intramuscularly once a day for 5 days. She showed improvement in feed intake after the third day. On 23.xi.04 bilateral epistaxis was marked and the animal went off-feed again. Styptovit tablets 2nos. (Adrenochrome monosemicarbazone; Dr. Reddy's Lab. Ltd., Hyderabad), Dexamethasone 2ml I/M (Dexona inj.; Cadila Health Care Ltd., Ahmedabad), Ranitidine 2ml I/M (Ranitin Inj. 2mg/ $\mathrm{ml}$; Torrent Pharma Ltd., Gujarat) and Neohepatex 5ml (Inj.; Biological Event, Medak, Hyderabad) were administered for two days with no improvement. On 25.xi.04 administration of Botropase 2ml I/M (Inj.; Jagat Pharma, Bangalore) reduced the epistaxis. On observation no abnormality in eye lid movement, eye balls or forehead contour could be marked. However, respiratory distress with snoring was prominent from both nostrils. As the animal relapsed back to inappetance and epistaxis, it was decided to tranquilize the tigress for physical and radiological examination of nasal cavity.

A mixture of $0.60 \mathrm{mg}$ of atropine sulphate, $150 \mathrm{mg}$ of Xylazine hydrochloride (Troy Lab., Australia) and 300mg of Ketamine hydrochloride (Troy Lab., Australia) was injected intramuscularly through a jab stick. The animal was recumbent within 10 minutes and transported to the zoo hospital. D5 (Dextrose 5\% inj.; Claris Life Sciences Ltd., Ahmedabad) was infused throughout the procedure. A sterile swab was collected from the nostril for culture and sensitivity. Physical examination revealed growths in both the nostrils and manipulation with finger tip resulted in profuse bleeding. The animal's head was kept lowered and the nostrils were irrigated with ice water to stop haemorrhage. An intra-oral dorso-ventral radiograph of the nasal area was taken using a mobile X-ray machine. RL 500ml (Ringer Lactate inj.; Claris Life Sciences Ltd., Ahmedabad), Rintose 500ml I/V (Wockhard Ltd., Mumbai); Botropase $2 \mathrm{ml}$ Inj. (Juggat Pharma, Ahmedabad), Kapilin 2ml (1ml. Inj.; Glaxo Smithkline Pharma Ltd., Nasik) and Cefotaxime 1g (Taxim Inj.; Alkem Laboratory Ltd., Mumbai) were administered intramuscularly. The animal was revived with $1 \mathrm{ml}$ of $\mathrm{I} / \mathrm{V}$ Yohimbine hydrochloride (Antagozil Inj.; Troy Lab., Australia). The radiograph revealed constricted nasal chambers with degenerative changes in the turbinates of the right side (Image $\left.1^{\mathrm{w}}\right)$. Cultural examination of nasal swab revealed the presence of gram -ve rods of Anthracoid baccilus and Staphylococci. The organisms were sensitive to Ofloxacin, Ciprofloxacin and
Moxifloxacin. Then antibiotic was changed to I/M Ciprofloxacin $80 \mathrm{mg}$ administered once daily for five days with spraying of Ciprofloxacin liquid to the nasal passage. The injection of intramuscular Botropase 2ml, Kapillin 2ml, Dexamethasone $2 \mathrm{ml}$ and neohepatex $5 \mathrm{ml}$ was continued for five days. The animal showed improvement with control of epistaxis, increased food intake and normal respiration with absence of snoring. The tigress was released to her enclosure on 16.xii.04 as she had an uneventful recovery.

In the present case intermittent inappetance and epistaxis prompted us to tranquilise the tigress for physical and radiological examination. Biopsy of the masses from nasal cavity could not be taken because of profuse bleeding. Spraying the lesion with sensitive antibiotic, fluid therapy, styptics and parenteral sensitive antibiotic helped in recovery of the animal. The radiological finding of destruction of turbinate might have occurred due to trauma with secondary infection. The severe tissue inflammation at the nasal chambers might have caused narrowing of the lumen resulting in snoring sound and respiratory difficulty which was controlled by steroids and appropriate antibiotics. Loss of appetite might have been due to inability to smell which commonly occurs as a result of trauma and inflammation of external nares as suggested by Nelson and Wykes (1985) in dogs and cats. Improvement in clinical condition did not warrant for the retranquilisation and manipulation of the tigress for evaluation of the treatment regimen.

The tigress is 18 years old having littered five times giving birth to six white and none normal coloured cubs and is a pride of the Zoo.

\section{REFERENCE}

Nelson, A.W. and P.M. Wykes (1985). Upper respiratory system, pp. 961. In: Text Book of Small Animal Surgery. $1^{\text {st }}$ edition. W.B. Saunders \& Company, Philadelphia.

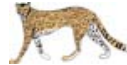

(c) Zoo Outreach Organisation; www.zoosprint.org 